\title{
Simultaneous Resource Accumulation and Payoff Allocation: A Cooperative Fuzzy Game Approach
}

\author{
Surajit Borkotokey ${ }^{D},{ }^{1}$ Rupok Neog, ${ }^{1}$ and Qiang Zhang ${ }^{2}$ \\ ${ }^{1}$ Department of Mathematics, Dibrugarh University, Dibrugarh 786004, India \\ ${ }^{2}$ School of Management and Economics, Beijing Institute of Technology, Beijing 100081, China \\ Correspondence should be addressed to Surajit Borkotokey; surajitbor@yahoo.com
}

Received 29 November 2017; Accepted 17 January 2018; Published 1 April 2018

Academic Editor: Pushpinder Singh

Copyright (c) 2018 Surajit Borkotokey et al. This is an open access article distributed under the Creative Commons Attribution License, which permits unrestricted use, distribution, and reproduction in any medium, provided the original work is properly cited.

\begin{abstract}
We develop a simultaneous resource accumulation and payoff allocation algorithm under the framework of a cooperative fuzzy game that builds on our earlier work on the role of satisfaction in resource accumulation and payoff allocation. The difference between the two models lies in the fact that while focus was more on getting an exact solution in our previous model, the negotiation process in the current model accounts more for the role of the intermediate stages. Moreover we characterize our solution using two properties: asymptotic fairness and efficiency. Our model includes a suitable penalty function to refrain players from unreasonable demands. We focus on real life situations where possibly one or more players compromise on their shares to ensure a binding agreement with the others.
\end{abstract}

\section{Introduction}

In the literature, cooperation among self-interested players under binding agreements has been well explained by the theory of crisp cooperative games. In [1-3] it is shown that players' satisfaction has the ability to set up a solution concept in a cooperative game which is easily computable through an algorithm. It is therefore important to take account of how coalitions are formed $v i s-\grave{a}-v i s$ and how the worth is allocated among participating players so that they are individually satisfied up to a desired level.

In this paper, we propose a dynamic process of accumulating resources from players and of finding a payoff allocation simultaneously under a cooperative fuzzy game theoretic environment. The preliminary works are done in [3]. Here we enhance the same model incorporating (a) the role of players in the intermediate steps of the negotiation process and (b) the notion of a penalty function that restrains the players from making unreasonable demands.

Consider, for example, the role of Uber and Ola cab services in different Indian cities. Both these companies acquire vehicles from the local vehicle providers of the cities on lease and offer them a portion of the profits accrued from the customers on per day basis. It follows that the cab services accumulate resource (in terms of number of vehicles from different service providers), generate some profit, and finally allocate this profit among the stack holders. It may be the case that the local vehicle providers have their shares in both the companies that results in different levels of satisfactions in terms of their trade relations with these companies. Retention becomes a big concern for both the companies. Therefore they need to satisfy the vehicle providers by providing sufficient opportunity to both generate and share the profit. More precisely let us consider a hypothetical situation where three agents $P_{1}, P_{2}$, and $P_{3}$ in their respective territorial areas collect monetary resources from the customers and provide this accumulated resource for an investment firm. The firm in turn invests the whole amount in shares of various companies (finitely many) in the market and rewards the agents according to their performances. The agents take notes on how their resources are being invested in different companies. This would determine how much payoff they would get after the firm profits from such investments. Here the manager of the firm acts as the mediator who 
takes care of all the resources of the agents. The payoffs to the agents are usually measured through their resource collection capacities, whereas, in addition to weighing on such straight performances, many organizations provide extra incentives adopting background corrections. This may include the geographical disadvantages the agents have to comply with in their territorial areas, their prospects towards future expansion of business, team working capabilities, and so on. Such incentives essentially gear up the satisfaction levels of the agents. However it is not found in the literature whether any standard procedure is followed in provision of such incentives. Moreover most of the business organizations follow the principle "if you perform well you get betteroff." Note that performance and satisfactions can never be universally standard. This motivates us to pursue the present study. Similar works can be traced back to [4-6] and so on.

In [7] a dynamic payoff allocation method that converges to a specific allocation under a given set of external restrictions is designed. Similar models of frequency allocations in wireless networks through a game in satisfaction form are found in [8] where payoff to a player accounts only for her satisfactions. However none of these studies uses the notion of fuzzy cooperative games as a tool to address their problems. For similar studies we refer to $[4,9,10]$ and so on.

In our previous model [3], the problem of simultaneous resource and payoff allocation among participating players by a mediator is discussed under a dynamic setup. It shows that an exact resource versus payoff allocation matrix evolves as a solution to a minimization problem at each stage of the negotiation process. However we observe that here the emphasis is put on how the exact solution (as a part of the limiting process) can be obtained without accounting for the intermediate solutions. In real life situations one needs to consider time and monetary constraints that do not allow the negotiation process to last longer. Therefore, in our present model we focus on the fact that the players get an optimal solution with the least possible time and money. We provide an axiomatic characterization of the exact solution which is based on two very natural axioms: efficiency and asymptotic fairness. We provide an example at the end of the paper to highlight this issue.

Let $N=\{1,2, \ldots, n\}$ be the set of players (agents) which is known as the grand coalition. Any subset of $N$ is called a crisp coalition. In a crisp coalition, a player can give either full participation (investment in our case) in the coalition with her complete resource or no participation (no resource). The collection of all crisp coalitions of $N$ is denoted by $2^{N}$. A crisp cooperative game is a pair $(N, v)$ where $v: 2^{N} \rightarrow$ $\mathbb{R}^{+} \cup\{0\}$ is a real valued function known as the characteristic function such that $v(\emptyset)=0$. If the players' set $N$ is fixed, the cooperative game is denoted by the function $v$. For each crisp coalition $c \in 2^{N}$, the real number $v(c)$ is known as the worth of the coalition $c$ (or profit incurred from $c$ ). On the other hand, if a player needs to participate in more than one coalition simultaneously, with her resources (or power) in hand, it is possible to provide only fractions of her full resource (power) for those coalitions. Such coalitions are called fuzzy coalitions. A fuzzy coalition is nothing but a fuzzy set of $N$, represented by an $n$-tuple, where its $i$ th component is the membership degree (or fraction of the power) of player $i$ ranging between 0 and 1 . A crisp coalition can be realized as a special type of fuzzy coalition where the degree of participation of any player in it is either 1 or 0. In the literature Aubin [11], Butnariu [12], and Branzei et al. [13] have well developed the theory of cooperative fuzzy games and justified the fuzzification in terms of the players' membership degrees in a coalition. In both crisp and fuzzy environment, it is quite necessary to determine how a fuzzy coalition structure is formed as well as how a suitable payoff distribution is proposed to the players accordingly. Solution concepts for cooperative games are found in [1117]. In [1], a new solution concept that evolves as a result of a dynamic negotiation process is defined. It is termed as the payoff allocation. In [2], a process of resource allocation of players is obtained in different cooperative actions with the formation of a fuzzy coalition structure. The key objective in $[1-3]$ is to investigate the influence of individual satisfactions upon a payoff and resource allocations (i.e., how it can be used to arrive at a suitable payoff and resource distribution over time). The process of resource allocation (equivalently resource accumulation) is made here synonymous with the formation of a fuzzy coalition. It follows that their model is equivalent to solving an $n$-person cooperative game with $m$ distinct fuzzy coalitions.

The goal of our present study is to provide a more developed and systematic treatment of satisfaction levels as a basis for negotiation among rational agents, who are capable of participating in different fuzzy coalitions with possibly varied rate of memberships simultaneously. We introduce the notion of a penalty to restrict the irrational demands of the players. Our present model is seen to be efficient than the one discussed in [3] in situations where the allocation process does not continue for long rather than stopping at some intermediate stage as part of a trade-off. We modify the variance function of [3] and show that at an intermediate stage our solution is more efficient than that of the earlier model.

The allocation process (Figure 1) goes exactly in the same way as that in [3]. Initially, the mediator would accumulate budgets/resources from the rational players. The mediator and the players would jointly determine the number of possible coalitions. Then the players would announce their expected total budgets/resources for each coalition. The mediator finds the optimal coalition structure and the optimal total budget allocation in such a way that the sum of all budgets allocated for all coalitions of the optimal coalition structure is equal to the total budget in her hand.

In the next stage of the allocation process, the mediator proposes the resource and payoff allocations simultaneously for each coalition of the optimal coalition structure which we call a solution here. The rational players would provide their satisfaction degrees in each coalition according to their own investment and payoff satisfaction functions. Based on this information, the mediator would update her belief and propose the next solution. The process continues until a stopping condition is reached. Thus the mediator would propose successive offers (solutions) to the players judging on 


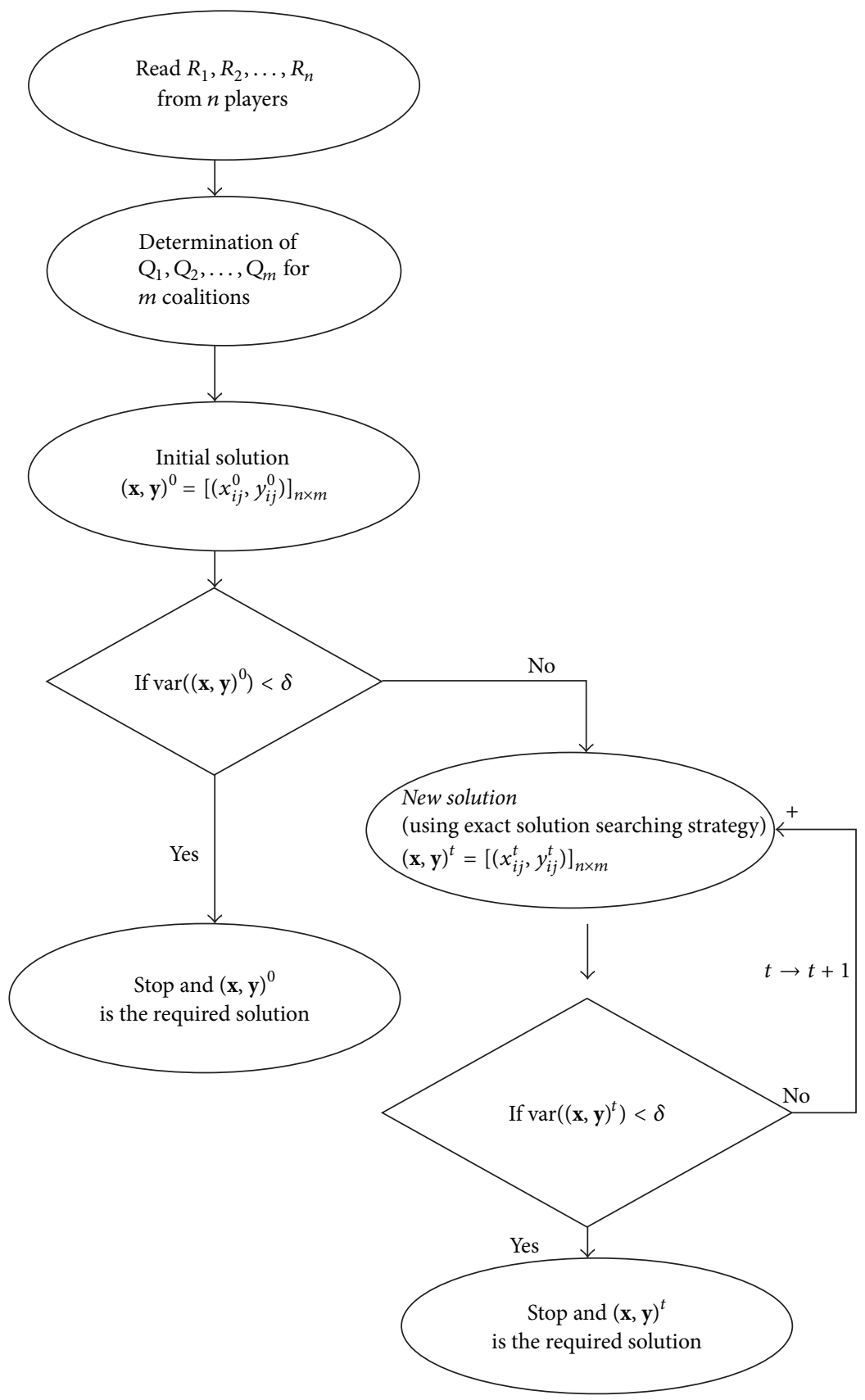

FIgURE 1: Flowchart of the allocation process.

their reactions to the previous proposal. Similar to what was proposed in [1-3] we construct a stopping rule and propose the process of updating the belief of the mediator by use of a pair of suitable functions towards the possible reactions of the players upon different offers of resource allocations and payoff allocations. We call them, respectively, the approximate investment satisfaction and approximate payoff satisfaction functions. A variance function is constructed to evaluate the closeness among the degrees of investment satisfaction and payoff satisfaction of the individual players in a coalition over a solution. If the variance of a solution is below a certain threshold to be determined by all the players collectively, then it would be considered as a possible trade-off solution to the problem. Situation may arise where the variance does not converge further after a particular threshold which is still an open problem and we keep it for our future works.

An exact solution is a solution whose variance is zero, see [3]. Therefore for an exact solution all the investment and payoff satisfactions are equal in each coalition. The process of proposing new allocations at successive stages leads to an exact solution. We assume that every player keeps her satisfaction functions unknown to the mediator and other players. The negotiation strategy is designed so that the mediator would propose only offers (possible solutions) for 
which the variance would be minimum at each stage of the negotiation process. In the negotiation process, each rational player has a single motive: to maximize her individual payoff, which is well represented by some monotonic increasing functions characterizing the fuzzy sets of their satisfactions. Since, negotiation requires a player to be considerate about the desires and views of all the other players. An appropriate negotiation process can restrain the players from making irrational demands while rewarding ones who are willing to work together by forming coalitions.

The remaining part of the paper is designed as follows. In Section 2, we build the theoretical framework of our proposed model and prove the existence of a better offer as a solution to our model. Section 3 deals with the notion of a penalty function to protect the negotiation processes from irrational demands. Some hypothetical examples are presented to discuss our model in Section 4. In Section 5, we present the concluding remarks. This section builds mainly on the notions and results discussed in [3]. We assume that for every $i(i=1,2,3, \ldots, n)$, the amount of resources available for agent $i$ is $R_{i} \geq 0$ (this can be time, money, etc.). In order to make the model simple we take the resource inputs and the payoff outputs of each player as real numbers. Each player $i$ can choose to invest in a joint project any portion $r_{i} \leq R_{i} \in \mathbb{R}$ of her total resource. We call this joint project a fuzzy coalition. The total resource allocation is represented by a nonnegative vector $\mathbf{R}=\left(R_{1}, \ldots, R_{n}\right) \in \mathbb{R}^{n}$, and possible coalitions are identified with the vectors that are (coordinatewise) smaller than $\mathbf{R}$. By what is called an abuse of notation we shall represent the sum $\sum_{i=1}^{n} R_{i}$ by $R$ here.

Thus formalizing the notion, we have the following:

for every nonnegative vector $\mathbf{R} \in \mathbb{R}^{n}$, let $F(\mathbf{R})$ be the box given by

$$
F(\mathbf{R})=\left\{\mathbf{r} \in \mathbb{R}^{n}: \mathbf{0} \leq \mathbf{r} \leq \mathbf{R}\right\} .
$$

The point $\mathbf{R}$ is interpreted as the "grand coalition" in fuzzy sense, and every $\mathbf{r} \in F(\mathbf{R})$ is a possible fuzzy coalition while $\mathbf{0} \in F(\mathbf{R})$ is the zero vector where all the players put zero resource. For every $\mathbf{R} \geq \mathbf{0}: \mathbf{R} \in \mathbb{R}^{n}$, a cooperative fuzzy game is a pair $(\mathbf{R}, v)$ such that

(i) $\mathbf{R} \in \mathbb{R}^{n}$ and $\mathbf{R} \geq \mathbf{0}$.

(ii) $v: F(\mathbf{R}) \rightarrow \mathbb{R}^{+} \cup\{0\}$ is bounded and satisfies $v(\mathbf{0})=0$.

The support of $\mathbf{r}=\left(r_{1}, r_{2}, \ldots, r_{n}\right) \in F(\mathbf{R})$ denoted by $\operatorname{supp}(\mathbf{r})$ is the set $\left\{i \in N \mid r_{i}>0\right\}$.

Since infinitely many fuzzy coalitions with limited resources of the players are not practically useful, let there be only $m$ possible fuzzy coalitions $(m<\infty)$. A fuzzy coalition structure $\mathbf{S}^{R}(m, n)=\left(\mathbf{S}_{1}^{n}(R), \mathbf{S}_{2}^{n}(R), \ldots, \mathbf{S}_{m}^{n}(R)\right)$ with respect to the grand coalition $\mathbf{R}$ (or equivalently $R$ ) is an $m$-vector whose components $\mathbf{S}_{j}^{n}(R), 1 \leq j \leq m$, with $n$ players are called fuzzy coalition variables. Thus a vector $\mathbf{r} \in F(\mathbf{R})$ is a fuzzy coalition in the fuzzy coalition structure $\mathbf{S}^{R}(m, n)$ if $\mathbf{S}_{j}^{n}(R)=\mathbf{r}$ for some $j: 1 \leq j \leq m$.

Note that if $v: F(\mathbf{R}) \rightarrow \mathbb{R}^{+} \cup\{0\}$ is continuous and all the resources are of same kind, then $v$ depends only on the total amount of resources $Q$ of a coalition $\mathbf{r} \in F(\mathbf{R})$ instead of different distributions: that is, $v$ is constant on every set $\left\{\left(r_{1}, r_{2}, \ldots, r_{n}\right) \mid \sum_{i=1}^{n} r_{i}=Q\right\}$ for each $Q \in \mathbb{R}$. For example, if resources are considered in monetary units, then $v$ being symmetric in all variables generates a unique function $\zeta: \mathbb{R} \rightarrow \mathbb{R}^{+} \cup\{0\}$ such that $v=\zeta$ o $\xi$ where $\xi: F(\mathbf{R}) \rightarrow$ $\mathbb{R}$ is defined for every $\mathbf{r} \in F(\mathbf{R})$ by $\xi(\mathbf{r})=\sum_{i=1}^{n} r_{i}$. Thus with an abuse of notations we can use $v$ and $\zeta$ alternatively and hence represent $v(\mathbf{r})$ by $v\left(\sum_{i}^{n} r_{i}\right)$. For every $j \in\{1,2,3, \ldots, m\}$, let the maximum resource that can be accumulated in the $j$ th fuzzy coalition be $Q_{j}$. In view of the above discussion a fuzzy coalition structure $\mathbf{S}^{R}(m, n)$ can therefore be denoted by an $m$-tuple of real numbers $\left(Q_{1}, Q_{2}, \ldots, Q_{m}\right)$. A fuzzy coalition structure $\left(Q_{1}, Q_{2}, \ldots, Q_{m}\right)$ is optimal if it maximizes $\sum_{i=1}^{m} v\left(Q_{j}\right)$. Here $v\left(Q_{j}\right)$ denotes $v\left(r_{1}, r_{2}, \ldots, r_{n}\right)$ where $\sum_{i=1}^{n} r_{i}=Q_{j}$. The remaining of the paper assumes that $v$ is continuous and the resources to be put in any coalition are of the same type.

Definition 1. A matrix $\mathbf{x}=\left(x_{i j}\right)_{i=1, j=1}^{n, m}$ of real entries with the conditions $\sum_{i=1}^{n} x_{i j}=Q_{j}$ and $\sum_{j=1}^{m} x_{i j}=R_{i}$ is called a resource investment matrix and the corresponding entries $x_{i j}$ are called resource investments.

It follows that a resource investment matrix is a fuzzy coalition structure.

Definition 2 (see [3]). A solution ( $\mathbf{x}, \mathbf{y})$ of a cooperative game $v$ with $n$ players and $m$ fuzzy coalitions is an $n \times m$ bimatrix, where the coordinates of the $(i, j)$ th entry $\left(x_{i j}, y_{i j}\right)$ represent the respective resource investments and payoff allocations to player $i$ in the $j$ th coalition, satisfying the following four conditions:

(i) $0 \leq x_{i j} \leq R_{i}, 0 \leq y_{i j} \leq v\left(Q_{j}\right), i=1,2, \ldots, n ; j=$ $1,2, \ldots, m$.

(ii) $\sum_{j=1}^{m} x_{i j}=R_{i}, i=1,2, \ldots, n$.

(iii) $\sum_{i=1}^{n} x_{i j}=Q_{j}, j=1,2, \ldots, m$.

(iv) $\sum_{i=1}^{n} y_{i j}=v\left(Q_{j}\right), j=1,2, \ldots, m$.

Let $\delta_{n, m}(v)$ denote the set of all solutions $(\mathbf{x}, \mathbf{y})$ with respect to a cooperative game $v$ with $n$ players and $m$ fuzzy coalitions. The column vectors $\mathbf{x}_{j}$ and $\mathbf{y}_{j}(j=1,2, \ldots, m)$ corresponding to a solution $(\mathbf{x}, \mathbf{y})$ represent, respectively, the resource investment and payoff allocation vectors for the $j$ th coalition.

1.1. Formation of the Cooperative Fuzzy Game. Initially, player $i \in N=\{1,2, \ldots, n\}$ would submit her budget $R_{i}$ to the mediator. The mediator and the players jointly determine an optimal coalition structure; see [2]. . $^{1}$

The mediator offers fractions of resources among the players and offers a possible investment vector to invest in a joint project (that is to form a fuzzy coalition). The players would react by announcing their degrees of satisfactions. Thus, we associate a satisfaction function for each player for such resource investment and call it the investment satisfaction. 
Thus the investment satisfaction function of player $i$ in the cooperative fuzzy game $v$, denoted by $I_{v}^{i}$, is defined as follows (see [3]).

Definition 3. Let $R_{i}$ be the total resource of the player $i \in N$ in a cooperative game with fuzzy coalitions $v$. Then the function $I_{v}^{i}: \mathbb{R} \rightarrow[0,1]$ is said to be an investment satisfaction function of player $i$ if the following hold:

(a) $I_{v}^{i}(x)=0$, when $x \leq 0 ; I_{v}^{i}(x)=1$, when $x \geq R_{i}$.

(b) $I_{v}^{i}$ is continuously differentiable and strictly monotonic increasing in $\left[0, R_{i}\right]$.

(c) $I_{v}^{i}$ is convex in $\left[0, R_{i}\right]$ (equivalently the derivative of $I_{v}^{i}$ is monotonic increasing in $\left.\left[0, R_{i}\right]\right)$.

Probable physical significance of the above assumptions as given in [1] is that each player $i$ is keen to invest her whole resource $R_{i}$. Her degree of satisfaction is therefore zero if she has no investment at all and one if she has full investment there. Moreover, it is natural to expect that satisfaction of any player increases continuously with respect to her investment. Furthermore, every player tries to increase her resource investment in a coalition, so the investment satisfaction functions are convex.

The payoff satisfaction function for player $i$ over her payoff allocation in the cooperative fuzzy game $v$, denoted by $P_{v}^{i}$, is defined as follows.

Definition 4. Let $d_{i}=v(R)-v\left(R_{-i}\right)$ where $R_{-i}$ is the total resource in some coalition where player $i$ 's investment is zero and each player $k$, such that $k \neq i$, invests $R_{k}$ in it. One can interpret $d_{i}$ as the fuzzy version of the marginal contribution of player $i$. Then the function $P_{v}^{i}: \mathbb{R} \rightarrow[0,1]$ is said to be a payoff satisfaction function of the player $i$ if the following hold:

(a) $P_{v}^{i}(x)=0$, when $x \leq 0 ; P_{v}^{i}(x)=1$, when $x \geq d_{i}$.

(b) $P_{v}^{i}$ is continuously differentiable and strictly monotonic increasing in $\left[0, d_{i}\right]$.

(c) $P_{v}^{i}$ is convex or equivalently the derivative of $P_{v}^{i}$ is monotonic increasing in $\left[0, d_{i}\right]$.

Probable physical significance of the above assumptions as given in [1] is that each player $i$ has a degree of satisfaction for any reward ranging from zero to $d_{i}$, where zero is the least reward player $i$ can assume and $d_{i}$ the maximum. Moreover, degree of satisfaction increases if the reward increases from 0 to $d_{i}$. So the function $P_{v}^{i}$ is considered as continuously differentiable and also strictly monotonic increasing in $\left[0, d_{i}\right]$. Again in any coalition the players mostly try to increase their payoffs by decreasing their satisfaction degrees upon an offer. Thus Assumption 3 reflects the choice of a particular satisfaction function that tries to address the individual preferences of each player.

Remark 5. Since the range of investment satisfaction and payoff satisfaction functions in Definitions 3 and 4 is normalized to $[0,1]$ the implicit assumption we make here is that the satisfaction of each player is a cardinal utility that admits an interpersonal comparison.

Definition 6 (see [3]). A solution (x,y) of a cooperative game $v$ is said to be an exact solution if all the players in every fuzzy coalition are equally satisfied with their resources and payoffs; that is,
(i) $I_{v}^{1}\left(x_{1 j}\right)=I_{v}^{2}\left(x_{2 j}\right)=\cdots=I_{v}^{n}\left(x_{n j}\right), j=1,2, \ldots, m$;
(ii) $P_{v}^{1}\left(y_{1 j}\right)=P_{v}^{2}\left(y_{2 j}\right)=\cdots=P_{v}^{n}\left(y_{n j}\right), j=1,2, \ldots, m$.

Definition 7 (see [3]). A solution ( $\mathbf{x}, \mathbf{y})$ of $v$ is said to be an approximate solution if there exist $k$ and $l(k \neq l)$ such that $I_{v}^{k}\left(x_{k j}\right) \neq I_{v}^{l}\left(x_{l j}\right)$ or $P_{v}^{k}\left(y_{k j}\right) \neq P_{v}^{l}\left(y_{l j}\right)$ for some $j \in\{1,2, \ldots$, $m\}$.

Let $\mathscr{H}$ be the history over which the mediator makes proposals to the players. Given a time (stage) $t \in \mathscr{H}$ and the set $\mathcal{S}_{n, m}^{t}(v)$ of solutions $\left(\mathbf{x}^{t}, \mathbf{y}^{t}\right)$, let us define the following sets [3]:

$$
\begin{aligned}
& A_{i 1}^{t}:=\left\{\mathbf{x}_{j}^{t} \in F(\mathbf{R}): I_{v}^{i}\left(x_{i j}^{t}\right)>\overline{I_{v}\left(\mathbf{x}_{j}^{t}\right)}\right\}, \\
& A_{i 2}^{t}:=\left\{\mathbf{x}_{j}^{t} \in F(\mathbf{R}): I_{v}^{i}\left(x_{i j}^{t}\right)<\overline{I_{v}\left(\mathbf{x}_{j}^{t}\right)}\right\}, \\
& A_{i 3}^{t}:=\left\{\mathbf{x}_{j}^{t} \in F(\mathbf{R}): I_{v}^{i}\left(x_{i j}^{t}\right)=\overline{I_{v}\left(\mathbf{x}_{j}^{t}\right)}\right\}, \\
& B_{i 1}^{t}:=\left\{\mathbf{y}_{j}^{t} \in \mathbb{R}^{n}: P_{v}^{i}\left(y_{i j}^{t}\right)>\overline{P_{v}\left(\mathbf{y}_{j}^{t}\right)}\right\}, \\
& B_{i 2}^{t}:=\left\{\mathbf{y}_{j}^{t} \in \mathbb{R}^{n}: P_{v}^{i}\left(y_{i j}^{t}\right)<\overline{P_{v}\left(\mathbf{y}_{j}^{t}\right)}\right\}, \\
& B_{i 3}^{t}:=\left\{\mathbf{y}_{j}^{t} \in \mathbb{R}^{n}: P_{v}^{i}\left(y_{i j}^{t}\right)=\overline{P_{v}\left(\mathbf{y}_{j}^{t}\right)}\right\},
\end{aligned}
$$

where $\overline{I_{v}\left(\mathbf{x}_{j}^{t}\right)}=\sum_{i=1}^{n} I_{v}^{i}\left(x_{i j}^{t}\right) / n$ and $\overline{P_{v}\left(\mathbf{y}_{j}^{t}\right)}=\sum_{i=1}^{n} P_{v}^{i}\left(y_{i j}^{t}\right) / n$.

For each $t \in \mathscr{H}$, let us call the solution $\left(\mathbf{x}^{t}, \mathbf{y}^{t}\right) \in \mathcal{S}_{n, m}^{t}(v)$ a dynamically evolved solution (DES) at time $t$.

\subsection{Our Model}

Definition 8. In order to obtain a better solution from the previous solution, we define the variance function var : $\mathcal{S}_{n, m}(v) \rightarrow \mathbb{R}$ as follows:

$$
\begin{aligned}
& \operatorname{var}(\mathbf{x}, \mathbf{y}) \\
& =\sqrt{\sum_{j=1}^{m} \sum_{i=1}^{n}\left\{\left(I_{v}^{i}\left(x_{i j}\right)-\overline{I_{v}\left(\mathbf{x}_{j}\right)}\right)^{2}+\overline{\left.\left(P_{v}^{i}\left(y_{i j}\right)-\overline{P_{v}\left(\mathbf{y}_{j}\right)}\right)^{2}+\gamma\right\}},\right.},
\end{aligned}
$$

where $\gamma=\left(I_{v}^{i}\left(x_{i j}\right) P_{v}^{i}\left(y_{i j}\right)-\overline{I_{v}\left(\mathbf{x}_{j}\right)} \overline{P_{v}\left(\mathbf{y}_{j}\right)}\right)^{2}$ and $\overline{I_{v}\left(\mathbf{x}_{j}\right)}$ and $\overline{P_{v}\left(\mathbf{y}_{j}\right)}$ are as given by Eq. (2).

Note that in (3), $\gamma$ relates to the satisfactions of the players with their resources and payoffs at each stage. In our model in [3], effect of $\gamma$ was implicit and could only be realized at the last stage of the exact solution searching procedure. In the intermediate stages the investment and the payoff satisfactions were assumed to be independent of one another. 
However in reality this is more of a strong assumption as satisfactions cannot be treated in isolation. Moreover, we have already explained that due to time and monetary constraints it is unlikely to allow the allocation process to iterate for long to reach the exact solution and rather a trade-off is sought to arrive at some optimal solution. Thus $\gamma$ suggests that optimal solutions at intermediate stages should also account for such interdependence between the investment and the payoff satisfaction functions.

Definition 9. A solution $(\mathbf{x}, \mathbf{y})$ is said to be a better approximate solution over another solution $\left(\mathbf{x}^{\prime}, \mathbf{y}^{\prime}\right)$ if $\operatorname{var}(\mathbf{x}, \mathbf{y})<$ $\operatorname{var}\left(\mathbf{x}^{\prime}, \mathbf{y}^{\prime}\right)$.

1.3. Negotiated Allocation Strategies. We assume that, in our allocation model, the negotiation is governed by the negotiation strategies adopted by the mediator. These strategies determine how the mediator identifies suitable solutions and how the players evaluate those solutions in the light of their own interests. The mediator would propose solutions at each stage/time of the negotiation process until a solution is accepted by the players with equal satisfactions within a coalition using exact solution searching strategy [3]. Alternatively the mediator may suggest the players to adopt a trade-off or an optimal solution searching strategy [3] which we discuss in the following briefly.

In the allocation process the mediator does not have any initial information about the investment and payoff satisfaction functions of the players; she will only update her belief on each player at each succeeding stage using the lower and upper bounds of those satisfactions. At stage $t$ of the allocation process, these bounds can be obtained for player $i \epsilon$ $N$ in the $j$ th coalition by defining two approximate functions (see [3] for more details); namely, $\lambda_{i j}^{t}: \mathbb{R} \rightarrow \mathbb{R}$ and $\mu_{i j}^{t}: \mathbb{R} \rightarrow$ $\mathbb{R}$. These functions are simply linear approximations (from below) of the actual satisfaction functions of the players for the next offer at stage $t+1$ and are obtained by joining the pairs of points $\left[(0,0),\left(x_{i j}^{t}, I_{v}^{i}\left(x_{i j}^{t}\right)\right)\right]$ and $\left[(0,0),\left(y_{i j}^{t}, P_{v}^{i}\left(y_{i j}^{t}\right)\right)\right]$, respectively. Thus we have

$$
\begin{aligned}
& \lambda_{i j}^{t}(x)=\frac{I_{v}^{i}\left(x_{i j}^{t}\right)}{x_{i j}^{t}} x, \quad \forall i \in N, j=1,2, \ldots, m, \\
& \mu_{i j}^{t}(y)=\frac{P_{v}^{i}\left(y_{i j}^{t}\right)}{y_{i j}^{t}} y, \quad \forall i \in N, j=1,2, \ldots, m .
\end{aligned}
$$

For $\mathbf{x}_{j}^{t} \in A_{i 1}^{t}$ denote

$$
\begin{aligned}
a_{i j}^{t} & =\overline{I_{v}\left(\mathbf{x}_{j}^{t}\right)} \times \frac{x_{i j}^{t}}{I_{v}^{i}\left(x_{i j}^{t}\right)}, \\
b_{i j}^{t} & =x_{i j}^{t} .
\end{aligned}
$$

Similarly for $\mathbf{x}_{j}^{t} \in A_{i 2}^{t}$ denote

$$
\begin{aligned}
a_{i j}^{t} & =x_{i j}^{t}, \\
b_{i j}^{t} & =\overline{I_{v}\left(\mathbf{x}_{j}^{t}\right)} \times \frac{x_{i j}^{t}}{I_{v}^{i}\left(x_{i j}^{t}\right)} .
\end{aligned}
$$

Finally for $\mathbf{x}_{j}^{t} \in A_{i 3}^{t}$ denote

$$
\begin{aligned}
& a_{i j}^{t}=x_{i j}^{t}, \\
& b_{i j}^{t}=x_{i j}^{t} .
\end{aligned}
$$

In a similar manner, denote for $\mathbf{y}_{j}^{t} \in B_{i 1}^{t}$

$$
\begin{aligned}
& a_{i j}^{\prime t}=\overline{P_{v}\left(\mathbf{y}_{j}^{t}\right)} \times \frac{y_{i j}^{t}}{P_{v}^{i}\left(y_{i j}^{t}\right)}, \\
& b_{i j}^{\prime t}=y_{i j}^{t} .
\end{aligned}
$$

For $\mathbf{y}_{j}^{t} \in B_{i 2}^{t}$ denote

$$
\begin{aligned}
& a_{i j}^{\prime t}=y_{i j}^{t}, \\
& b_{i j}^{\prime t}=\overline{P_{v}\left(\mathbf{y}_{j}^{t}\right)} \times \frac{y_{i j}^{t}}{P_{v}^{i}\left(y_{i j}^{t}\right)} .
\end{aligned}
$$

Finally for $\mathbf{y}_{j}^{t} \in B_{i 3}^{t}$ denote

$$
\begin{aligned}
& a_{i j}^{\prime t}=y_{i j}^{t}, \\
& b_{i j}^{\prime t}=y_{i j}^{t} .
\end{aligned}
$$

Approximations of the satisfaction functions are shown in Figures 2(a), 2(b), 3(a), and 3(b).

Thus the expected proposal $\left(\mathbf{x}^{*}, \mathbf{y}^{*}\right)$ that benefits all the players can be defined as follows.

Definition 10. Assuming that the mediator offers a DES $\left(\mathbf{x}^{t}, \mathbf{y}^{t}\right)$ to the players at stage $t$ and that all the players subsequently announce their investment and payoff satisfaction degrees, the expected better resource allocation $\left(\mathbf{x}^{*}, \mathbf{y}^{*}\right)$ for the proposal at stage $t+1$ is defined as

$$
\left(\mathbf{x}^{*}, \mathbf{y}^{*}\right)=\arg \min _{(\mathbf{x}, \mathbf{y})}\{\sqrt{\alpha}: B\}
$$

where

$$
\alpha=\sum_{j=1}^{m} \sum_{i=1}^{n} \sum_{k=i}^{n}\left\{\left(\lambda_{i j}^{t}\left(x_{i j}\right)-\lambda_{k j}^{t}\left(x_{k j}\right)\right)^{2}+\left(\mu_{i j}^{t}\left(y_{i j}\right)-\mu_{k j}^{t}\left(y_{k j}\right)\right)^{2}+\left(\lambda_{i j}^{t}\left(x_{i j}\right) \mu_{i j}^{t}\left(y_{i j}\right)-\lambda_{k j}^{t}\left(x_{k j}\right) \mu_{k j}^{t}\left(y_{k j}\right)\right)^{2}\right\}
$$




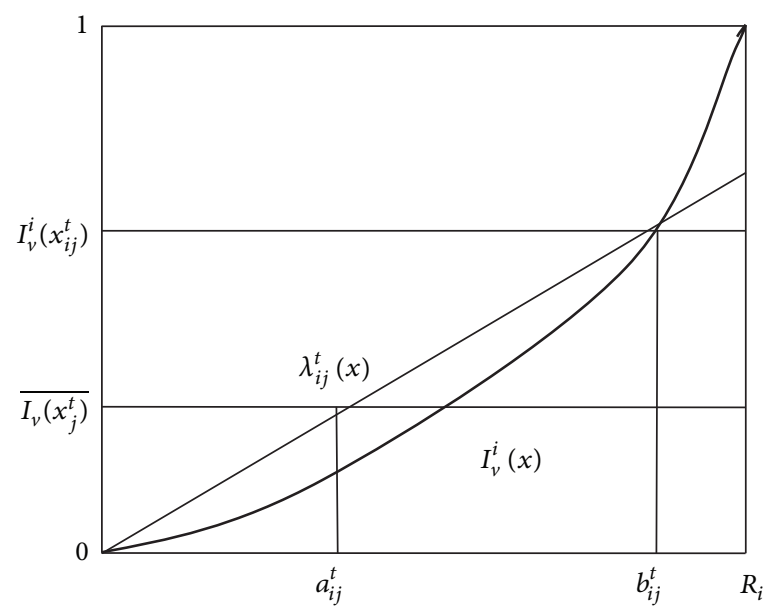

(a)

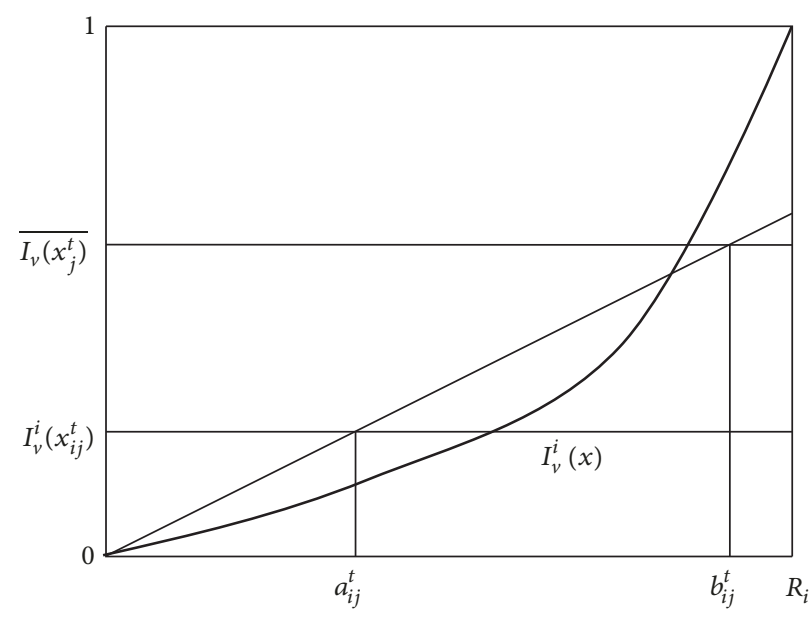

(b)

Figure 2

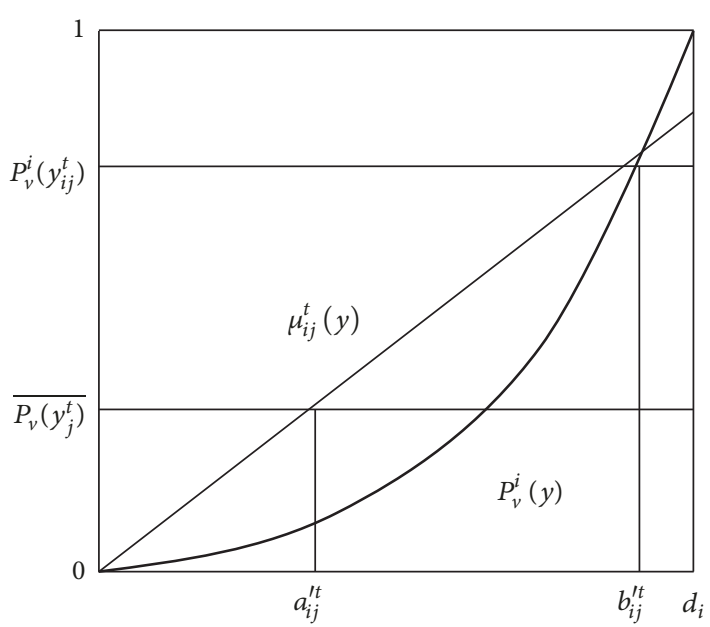

(a)

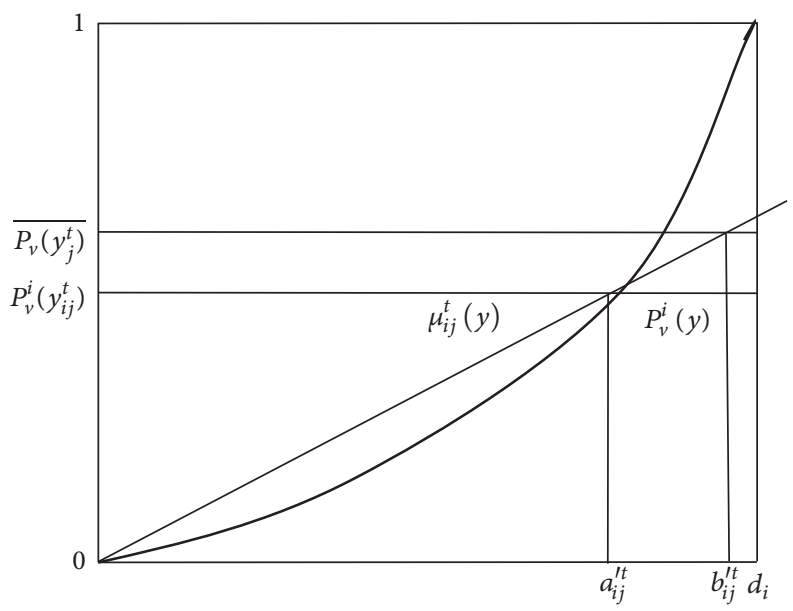

(b)

Figure 3

and the set of constraints $B$ is given by

$$
\begin{aligned}
B & =\left\{\sum_{i=1}^{n} x_{i j}=Q_{j}, \sum_{j=1}^{m} x_{i j}=R_{i}, a_{i j}^{t} \leq x_{i j} \leq b_{i j}^{t}, a_{i j}^{\prime t} \leq y_{i j}\right. \\
& \leq b_{i j}^{\prime t}, \sum_{i=1}^{n} y_{i j}=v\left(\mathbf{x}_{j}\right), i=1,2, \ldots, n, j \\
& =1,2, \ldots, m\} .
\end{aligned}
$$

Here $\alpha$ is the sum of the squared differences of different satisfaction levels of the players using approximate satisfaction functions in each fuzzy coalition. If at stage $t$, the proposed DES $\left(\mathbf{x}^{t}, \mathbf{y}^{t}\right)$ is such that, for some player $i$, any one of $A_{i 1}^{t}, A_{i 2}^{t}, B_{i 1}^{t}$, and $B_{i 2}^{t}$ is empty, then the exact solution searching process fails. In such case, if there exists an all player-accepted trade-off threshold $\delta$ such that $\operatorname{var}\left(\mathbf{x}^{t}, \mathbf{y}^{t}\right)<$ $\delta$, the current solution is accepted as an optimal solution. Otherwise the allocation process stops there and we say that the process ends with conflict.

Step 1. All the players will submit their resource $R_{i}, i=$ $1,2,3, \ldots, n$, to the mediator. Also, the players will construct their investment as well as payoff satisfaction functions independently using the cooperative game $v$.

Step 2. The mediator along with the players will determine the optimal coalition structure $\left\{Q_{1}, Q_{2}, \ldots, Q_{m}\right\}$ where $Q_{j}$ is the total budget for the coalition $j$.

Step 3. Here the mediator proposes the initial solution $\left(\mathbf{x}^{0}, \mathbf{y}^{0}\right)=\left[\left(x_{i j}^{0}, y_{i j}^{0}\right)\right]_{m \times n}$. Also, the players will show their satisfaction degree over their investments and payoffs. 
Step 4. The players determine a well accepted threshold $\delta$ and the mediator will check whether variance $\operatorname{var}\left(\mathbf{x}^{0}, \mathbf{y}^{0}\right)<$ $\delta$ or not. If yes she will stop the allocation process with $\left(\mathbf{x}^{0}, \mathbf{y}^{0}\right)$ as required solution. If no she will adopt the exact solution searching strategy if all the sets $A_{i 1}^{t}, A_{i 2}^{t}, B_{i 1}^{t}$, and $B_{i 2}^{t}$, $t=0,1,2, \ldots$, are nonempty. The mediator will approximate satisfaction functions by (4). Using these functions she will determine the bounds $a_{i j}^{t}, b_{i j}^{t}$, and $a_{i j}^{\prime t}, b_{i j}^{\prime t}, t=0,1,2, \ldots$, to be used in the minimization of (11) for the next proposal $\left(\mathbf{x}^{t+1}, \mathbf{y}^{t+1}\right)=\left[\left(x_{i j}^{t+1}, y_{i j}^{t+1}\right)\right]_{m \times n}$. If any one of the four sets $A_{i 1}^{t}, A_{i 2}^{t}, B_{i 1}^{t}$, and $B_{i 2}^{t}, t=0,1,2, \ldots$, is empty, the allocation process will stop with conflict.

Step 5. The mediator proposes $\left(\mathbf{x}^{t+1}, \mathbf{y}^{t+1}\right)=\left[\left(x_{i j}^{t+1}, y_{i j}^{t+1}\right)\right]_{m \times n}$, using minimization, (11) will repeat Step 4 to get an exact solution with variance 0 or optimal solution with variance less than $\delta$ (nonzero).

Lemma 11. Let the DES at stage $t$ be $\left(\mathbf{x}^{t}, \mathbf{y}^{t}\right)=\left[\left(x_{i j}^{t}, y_{i j}^{t}\right)\right]_{m \times n}$ such that for each player $i$, none of $A_{i 1}^{t}, A_{i 2}^{t}, B_{i 1}^{t}$ and $B_{i 2}^{t}$ is empty. Let the respective degrees of investment and payoff satisfactions given by player $i$ be $I_{v}^{i}\left(x_{i j}^{t}\right)$ and $P_{v}^{i}\left(y_{i j}^{t}\right)$; then we must have

$$
\begin{gathered}
\sum_{\mathbf{x}_{j}^{t} \in A_{i 1}^{t}} x_{i j}^{t}>\sum_{\mathbf{x}_{j}^{t} \in A_{i 1}^{t}} a_{i j}^{t}, \\
\sum_{\mathbf{x}_{j}^{t} \in A_{i 2}^{t}} b_{i j}^{t}>\sum_{\mathbf{x}_{j}^{t} \in A_{i 2}^{t}} x_{i j}^{t}, \\
\sum_{i \in A_{1 j}^{t}} x_{i j}^{t}>\sum_{i \in A_{1 j}^{t}} a_{i j}^{t}, \\
\sum_{i \in A_{2 j}^{t}} b_{i j}^{t}>\sum_{i \in A_{2 j}^{t}} x_{i j}^{t}, \\
\sum_{\mathbf{y}_{j}^{t} \in B_{i 1}^{t}} y_{i j}^{t}>\sum_{\mathbf{y}_{j}^{t} \in B_{i 1}^{t}} a_{i j}^{\prime t}, \\
\sum_{\mathbf{y}_{j}^{t} \in B_{i 2}^{t}} b_{i j}^{\prime t}>\sum_{\mathbf{y}_{j}^{t} \in A_{i 2}^{t}} y_{i j}^{t}, \\
\sum_{i \in B_{1 j}^{t}} y_{i j}^{t}>\sum_{i \in B_{1 j}^{t}} a_{i j}^{\prime t}, \\
\sum_{i \in B_{2 j}^{t}} b_{i j}^{\prime t}>\sum_{i \in B_{2 j}^{t}} y_{i j}^{t},
\end{gathered}
$$

where

$$
\begin{aligned}
& A_{1 j}^{t}=\left\{i \in N: I_{v}^{i}\left(x_{i j}^{t}\right)>\overline{I_{v}\left(\mathbf{x}_{j}^{t}\right)}\right\}, \\
& A_{2 j}^{t}=\left\{i \in N: I_{v}^{i}\left(x_{i j}^{t}\right)<\overline{I_{v}\left(\mathbf{x}_{j}^{t}\right)}\right\}, \\
& B_{1 j}^{t}=\left\{i \in N: P_{v}^{i}\left(y_{i j}^{t}\right)>\overline{P_{v}\left(\mathbf{y}_{j}^{t}\right)}\right\}, \\
& B_{2 j}^{t}=\left\{i \in N: P_{v}^{i}\left(y_{i j}^{t}\right)<\overline{P_{v}\left(\mathbf{y}_{j}^{t}\right)}\right\} .
\end{aligned}
$$

Proof. The proof follows immediately from Lemma 1 in [3] and therefore omitted here.

Theorem 12. Let $v \in G_{F}(\mathbf{R})$. At stage $t$, let $\left(\mathbf{x}^{t}, \mathbf{y}^{t}\right)=$ $\left[\left(x_{i j}^{t}, y_{i j}^{t}\right)\right]_{m \times n}$ be a DES which is not exact, and for each $i$, none of the sets $A_{i 1}^{t}, A_{i 2}^{t}, B_{i 1}^{t}$, and $B_{i 2}^{t}$ is empty.

Then there exists at least one better DES at stage $t+1$, $\left(\mathbf{x}^{t+1}, \mathbf{y}^{t+1}\right)=\left[\left(x_{i j}^{t+1}, y_{i j}^{t+1}\right)\right]_{m \times n}$ such that

(a) for $\mathbf{x}_{j}^{t} \in A_{i 1}^{t}, a_{i j}^{t} \leq x_{i j}^{t+1} \leq b_{i j}^{t}$, where $a_{i j}^{t}$ and $b_{i j}^{t}$ are given by (5);

(b) for $\mathbf{x}_{j}^{t} \in A_{i 2}^{t}, a_{i j}^{t} \leq x_{i j}^{t+1} \leq b_{i j}^{t}$, where $a_{i j}^{t}$ and $b_{i j}^{t}$ are given by (6);

(c) for $\mathbf{y}_{j}^{t} \in B_{i 1}^{t}, a_{i j}^{\prime t} \leq y_{i j}^{t+1} \leq b_{i j}^{\prime t}$, where $a_{i j}^{\prime t}$ and $b_{i j}^{\prime t}$ are given by (8);

(d) for $\mathbf{y}_{j}^{t} \in B_{i 2}^{t}, a_{i j}^{\prime t} \leq y_{i j}^{t+1} \leq b_{i j}^{\prime t}$, where $a_{i j}^{\prime t}$ and $b_{i j}^{\prime t}$ are given by (9).

Proof. We have to prove that there exists a better DES at stage $t+1$ over the one at stage $t$. This is achieved in two steps. In Step 1 we prove the existence of such a solution to the game, and then in Step 2, we show that this solution is better than the previous one.

Step 1 (existence of a solution). This part follows directly from Theorem 1 in [3] and so is omitted here.

Step 2 (there is a new DES better than the previous one). For $\mathbf{x}_{j}^{t} \in A_{i 1}^{t}, I_{v}^{i}\left(x_{i j}^{t}\right)>\overline{I_{v}\left(\mathbf{x}_{j}^{t}\right)}$; for $\mathbf{x}_{j}^{t} \in A_{i 2}^{t}, I_{v}^{i}\left(x_{i j}^{t}\right)<\overline{I_{v}\left(\mathbf{x}_{j}^{t}\right)}$; for $i \in A_{1 j}^{t}, I_{v}^{i}\left(x_{i j}^{t}\right)>\overline{I_{v}\left(\mathbf{x}_{j}^{t}\right)}$; and for $i \in A_{2 j}^{t}, I_{v}^{i}\left(x_{i j}^{t}\right)<\overline{I_{v}\left(\mathbf{x}_{j}^{t}\right)}$; Thus, we can select an $\mathbf{x} \in \mathbb{R}^{n} \times F(\mathbf{R})$ such that $a_{i j}^{t} \leq x_{i j} \leq b_{i j}^{t}$ for all $i$ and $j ; \sum_{j=1}^{m} a_{i j}^{t} \leq \sum_{j=1}^{m} x_{i j}=R_{i} \leq \sum_{j=1}^{m} b_{i j}^{t}$ for each $i$; and $\sum_{i=1}^{n} a_{i j}^{t} \leq \sum_{i=1}^{n} x_{i j}=Q_{j} \leq \sum_{i=1}^{n} b_{i j}^{t}$ for all $j$. Here we are using the fact that $\sum_{i=1}^{n} R_{i}=\sum_{j=1}^{m} Q_{j}$, along with the following:

(i) for some player $i$ and some $\mathbf{x}_{j}^{t} \in A_{i 1}^{t}, I_{v}^{i}\left(x_{i j}\right)<I_{v}^{i}\left(x_{i j}^{t}\right)$,

(ii) for some player $i$ and some $\mathbf{x}_{j}^{t} \in A_{i 2}^{t}, I_{v}^{i}\left(x_{i j}\right)>I_{v}^{i}\left(x_{i j}^{t}\right)$.

Similarly, one can find a $y \in \mathbb{R}^{n} \times \mathbb{R}^{m}$ using the following facts:

(i) for $\mathbf{y}_{j}^{t} \in B_{i 1}^{t}, P_{v}^{i}\left(y_{i j}^{t}\right)>\overline{P_{v}\left(\mathbf{y}_{j}^{t}\right)}$;

(ii) for $\mathbf{y}_{j}^{t} \in B_{i 2}^{t}, P_{v}^{i}\left(y_{i j}^{t}\right)<\overline{P_{v}\left(\mathbf{y}_{j}^{t}\right)}$;

(iii) for $i \in B_{1 j}^{t}, P_{v}^{i}\left(y_{i j}^{t}\right)>\overline{P_{v}\left(\mathbf{y}_{j}^{t}\right)}$;

(iv) for $i \in B_{2 j}^{t}, P_{v}^{i}\left(y_{i j}^{t}\right)<\overline{P_{v}\left(\mathbf{y}_{j}^{t}\right)}$.

Thus we have

$$
\begin{gathered}
\sum_{j=1}^{m} \sum_{i=1}^{n} \sum_{k=i}^{n}\left(I_{v}^{i}\left(x_{i j}\right)-I_{v}^{i}\left(x_{k j}\right)\right)^{2} \\
+\left(P_{v}^{i}\left(y_{i j}\right)-P_{v}^{i}\left(y_{k j}\right)\right)^{2}
\end{gathered}
$$




$$
\begin{aligned}
&+\left(I_{v}^{i}\left(x_{i j}\right) P_{v}^{i}\left(y_{i j}\right)-I_{v}^{i}\left(x_{k j}\right) P_{v}^{i}\left(y_{k j}\right)\right)^{2} \\
&< \sum_{j=1}^{m} \sum_{i=1}^{n} \sum_{k=i}^{n}\left(I_{v}^{i}\left(x_{i j}^{t}\right)-I_{v}^{i}\left(x_{k j}^{t}\right)\right)^{2} \\
&+\left(P_{v}^{i}\left(y_{i j}^{t}\right)-P_{v}^{i}\left(y_{k j}^{t}\right)\right)^{2} \\
&+\left(I_{v}^{i}\left(x_{i j}^{t}\right) P_{v}^{i}\left(y_{i j}^{t}\right)-I_{v}^{i}\left(x_{k j}^{t}\right) P_{v}^{i}\left(y_{k j}^{t}\right)\right)^{2} \Longrightarrow \\
& \sum_{j=1}^{m} \sum_{i=1}^{n} \sum_{k=i}^{n}\left(I_{v}^{i}\left(x_{i j}\right)-\overline{I_{v}\left(\mathbf{x}_{j}\right)}\right)^{2}+\left(P_{v}^{i}\left(y_{i j}\right)-\overline{P_{v}\left(\mathbf{y}_{j}\right)}\right)^{2} \\
&+\left(I_{v}^{i}\left(x_{i j}\right) P_{v}^{i}\left(y_{i j}\right)-\overline{I_{v}\left(\mathbf{x}_{j}\right) P_{v}\left(\mathbf{y}_{j}\right)}\right)^{2} \\
&< \sum_{j=1}^{m} \sum_{i=1}^{n} \sum_{k=i}^{n}\left(I_{v}^{i}\left(x_{i j}^{t}\right)-\overline{I_{v}\left(\mathbf{x}_{j}^{t}\right)}\right)^{2} \\
&+\left(P_{v}^{i}\left(y_{i j}^{t}\right)-\overline{P_{v}\left(\mathbf{y}_{j}^{t}\right)}\right)^{2} \\
&+\left(I_{v}^{i}\left(x_{i j}^{t}\right) P_{v}^{i}\left(y_{i j}^{t}\right)-\overline{I_{v}\left(\mathbf{x}_{j}^{t}\right) P_{v}\left(\mathbf{y}_{j}^{t}\right)}\right)^{2} \Longrightarrow \\
& \operatorname{var}(\mathbf{x}, \mathbf{y}) \leq \operatorname{var}\left(\mathbf{x}^{t}, \mathbf{y}^{t}\right) .
\end{aligned}
$$

Therefore, $(\mathbf{x}, \mathbf{y})$ is a better solution than $\left(\mathbf{x}^{t}, \mathbf{y}^{t}\right)$, so either we can denote $(\mathbf{x}, \mathbf{y})$ by $\left(\mathbf{x}^{t+1}, \mathbf{y}^{t+1}\right)$ or we can find better DES $\left(\mathbf{x}^{t+1}, \mathbf{y}^{t+1}\right)$ which will minimize $\operatorname{var}(\mathbf{x}, \mathbf{y})$. This completes the proof.

Theorem 13. If at each staget, for each player $i$, none of the sets $A_{1 i}^{t}, A_{2} i^{t}, B_{1} i^{t}$, and $B_{2} i^{t}$ is empty, then the process of obtaining a better DES converges to the exact solution.

Proof. The proof proceeds exactly in the same way as of Theorem 2 in [3] and so is omitted here.

\section{Axiomatization of the Exact Solution}

It follows from Theorem 13 above that the notion of an exact solution represents fairness and egalitarianism among players and the mediator is assumed to minimize the deviation from the fairness criterion. It is natural to ask: What should be a player's ultimate interest? Investment satisfaction or payoff satisfaction? The negotiated allocation strategies are designed so that the deviations in both investment and payoff satisfactions are minimized simultaneously. Thus a formal characterization of the solution to rationalize these strategies is equally important. In this section, we provide an axiomatic characterization of the exact solution with the axioms of efficiency and asymptotic fairness as mentioned in Section 1. In what follows, we provide the formal descriptions of these axioms.

Definition 14. A solution $\left(\mathbf{x}^{*}, \mathbf{y}^{*}\right)$ to the cooperative fuzzy game $v \in G_{F}(\mathbf{R})$ is called asymptotically fair if there exists a sequence of DES $\left(\mathbf{x}^{t}, \mathbf{y}^{t}\right)$ for $t \in \mathscr{H}$ and a continuous function $f: \bigcup_{t=0}^{\infty} \delta_{n, m}^{t}(v) \rightarrow \mathbb{R}$ such that

$$
\lim _{t \rightarrow \infty} f\left(\mathbf{x}^{t}, \mathbf{y}^{t}\right)=0=f\left(\mathbf{x}^{*}, \mathbf{y}^{*}\right) .
$$

It follows form Definition 14 that, when a solution is asymptotically fair, some kind of equitability among the players is preserved. The continuous function $f$ determines the type of equitable condition to be met by the solution.

Asymptotic Fairness (AF). A solution $\left(\mathbf{x}^{*}, \mathbf{y}^{*}\right)$ to the cooperative fuzzy game $v \in G_{F}(\mathbf{R})$ is asymptotically fair.

Efficiency $(E)$. For $(\mathbf{x}, \mathbf{y}) \in \mathcal{S}_{n, m}(v)$ of $v \in G_{F}(\mathbf{R})$ it holds that

$$
\begin{aligned}
& \text { (a) } \sum_{i=1}^{m} \sum_{j=1}^{n} x_{i j}=R \\
& \text { (b) } \sum_{i=1}^{m} \sum_{j=1}^{n} y_{i j}=v(\mathbf{R}) .
\end{aligned}
$$

Note that the efficiency axiom $E$ conforms with our basic assumption in the current model that accounts for accumulation of resource and allocation of payoffs together. Thus we have the following theorem.

Theorem 15. There exists an efficient and asymptotically fair solution to any game $v \in G_{F}(\mathbf{R})$. It is uniquely determined by the continuous function $f$ given by (17). In particular when $f \equiv$ var given by (3), it is the exact solution that is both efficient and asymptotically fair.

Proof. The proof follows from Lemma 11 and Theorems 12 and 13.

\section{Penalty}

Let us now introduce the notion of penalty to the negotiation process. This makes the allocation model more realistic. Usually, it happens that some players expect unreasonably high payoff by contributing merely in a coalition. This would render a never ending process of negotiations with little progress. In order to avoid such situations, the mediator may impose some penalty on the players who ask for unreasonable payoffs by showing unfair satisfaction against offers. We start with few definitions.

Definition 16. An investment satisfaction function for player $i \in N$ is said to be normal if its curve of description is a straight line, passing through the points $(0,0)$ and $\left(R_{i}, 1\right)$, with $R_{i} \neq 0$.

Thus, the equation of a normal investment satisfaction function for any player $i$ is given by

$$
I N_{v}^{i}(x)=\frac{x}{R_{i}} ; \quad \text { whenever } R_{i} \neq 0 .
$$

Definition 17. A payoff satisfaction function for player $i \in N$ is said to be normal if its curve is a straight line, passing through the points $(0,0)$ and $\left(d_{i}, 1\right)$, with $d_{i} \neq 0$. 


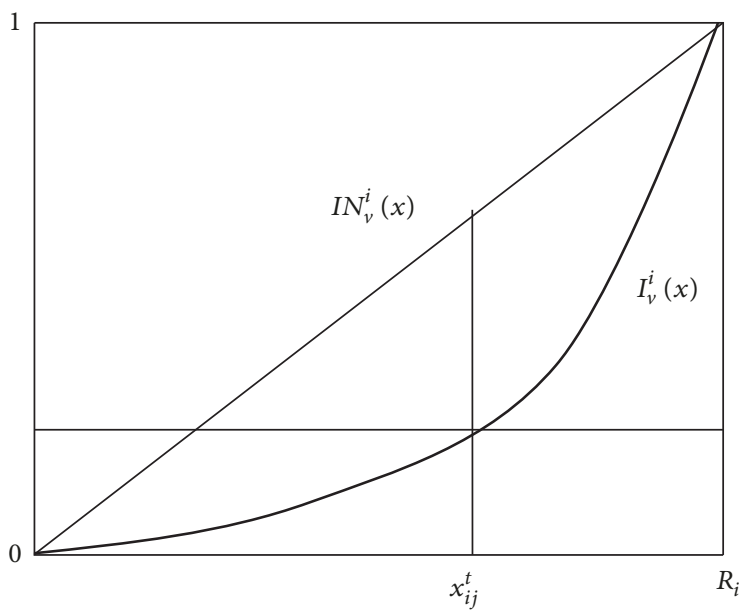

(a)

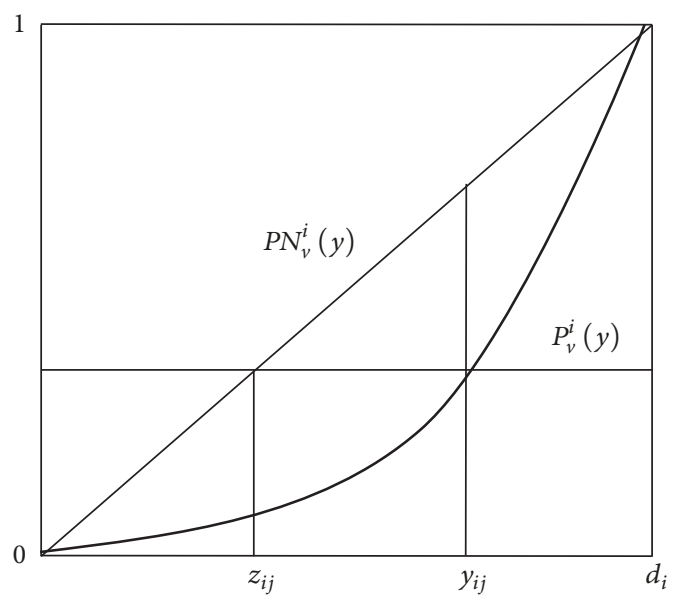

(b)

Figure 4

Thus, the equation of a normal payoff satisfaction function for any player $i$ is given by

$$
P N_{v}^{i}(x)=\frac{x}{d_{i}} ; \quad \text { whenever } d_{i} \neq 0 .
$$
game.

In what follows, we define the penalty function for our

Definition 18. Let $v \in G_{F}(\mathbf{R})$ and $(\mathbf{x}, \mathbf{y}) \in \delta_{n, m}(v)$. The penalty function of a player $i$ for the investment $x_{i j}$ and corresponding payoff $y_{i j}$ in the $j$ th coalition, with degrees of satisfaction $I_{v}^{i}\left(x_{i j}\right)$ and $P_{v}^{i}\left(y_{i j}\right)$, is defined by $p_{i}\left(x_{i j}, y_{i j}\right)=$ $\left(y_{i j} / d_{i}-P_{v}^{i}\left(y_{i j}\right)\right)-\left(x_{i j} / R_{i}-I_{v}^{i}\left(x_{i j}\right)\right)\left(1-c_{i j}\right)\left(y_{i j}-z_{i j}\right)$ whenever $0 \neq\left(y_{i j} / d_{i}-P_{v}^{i}\left(y_{i j}\right)\right) \geq\left(x_{i j} / R_{i}-I_{v}^{i}\left(x_{i j}\right)\right) \neq 0$ and $c_{i j} \neq 1$, $p_{i}\left(x_{i j}, y_{i j}\right)=0$, otherwise, where $c_{i j}=x_{i j} / R_{i}$ and $z_{i j}$ is such that $P_{v}^{i}\left(y_{i j}\right)=P N_{v}^{i}\left(z_{i j}\right)$.

In Figures 4(a) and 4(b), we have given an illustration of how penalty prevents the players from sticking to irrational expectations. In Figure 4(a), $I_{v}^{i}(\cdot)$ denotes the curve of actual investment satisfaction function for player $i$ while $I N_{v}^{i}(\cdot)$ denotes her normal investment satisfaction function as envisaged by the mediator. Similarly in Figure 4(b), $P_{v}^{i}(\cdot)$ denotes the curve of actual payoff satisfaction function for player $i$ while $P N_{v}^{i}(\cdot)$ denotes her normal payoff satisfaction function. Take two points $y_{i j}$ and $z_{i j}$ on the payoff axis, such that $P_{v}^{i}\left(y_{i j}\right)=P N_{v}^{i}\left(z_{i j}\right)$. Here, $\left(y_{i j} / d_{i}-P_{v}^{i}\left(y_{i j}\right)\right)$ is the difference of satisfactions due to player $i$ 's adoption of $P_{v}^{i}(\cdot)$ instead of $P N_{v}^{i}(\cdot)$ at $x_{i}$. Thus, from the definition of the penalty function the following are clear:

(a) Penalty of a player with lower participation rate will be more than that of another player with higher participation rate, when their satisfactions to a particular offer are equal.

(b) If two players with equal rates of participation try to increase their payoffs by lowering their satisfactions, then the penalty of player with lower satisfaction will be more than the penalty of the player with higher satisfaction.

(c) The penalty of player $i$ with full participation is zero; that is, the penalty of the player $i$ will be zero if $c_{i j}=1$.

(d) The penalty of player $i$ with normal payoff satisfaction function is zero.

(e) The penalty of player $i$ with normal investment satisfaction function is maximum. Thus the actual payoff received by player $i$ is given by $\sum_{j=1}^{m}\left(y_{i j}-p_{i}\left(x_{i j}, y_{i j}\right)\right)$.

\section{Examples}

Continuing the motivating example of the resource investment problem discussed in the Introduction, we now illustrate our model using some numerical calculations. In what follows we show that our model is better suitable for tradeoff and optimal strategies than the one discussed in [3]. We take two variations of the example. Let there be three types of shares $S_{1}, S_{2}$, and $S_{3}$ for the first variation and only two shares $S_{1}$ and $S_{2}$ for the second.

In each of these two examples, the manager of the firm invests on these shares after accumulating monetary resources from the agents. The first example illustrates the process of getting an exact solution while the second illustrates the use of penalty in the allocation process.

Example 1. Let all denominations be in a fixed multiple of some monetary units. Let the available monetary resources supplied by the agents be $R_{1}=10, R_{2}=15$, and $R_{3}=$ 20 , respectively (i.e., $\mathbf{R}=(10,15,20))$, and the sufficient monetary resources to be invested in each share (coalition) be $Q_{1}=10, Q_{2}=15$, and $Q_{3}=20$, respectively. Define the cooperative game $v: F(\mathbf{R}) \rightarrow \mathbb{R}^{+} \cup\{0\}$ assigning to every investment vector $\mathbf{x}=\left(x_{1}, x_{2}, x_{3}\right)$ a real number representing the return from the investments in those three shares by 


$$
v\left(\left(x_{1}, x_{2}, x_{3}\right)\right)= \begin{cases}\frac{\left(\sum_{i=1}^{3} x_{i}\right)^{2}}{5} & \text { if } \sum_{i=1}^{3} x_{i} \geq 5 \\ \sum_{i=1}^{3} x_{i} & \text { if } 0 \leq \sum_{i=1}^{3} x_{i} \leq 5 \\ 0 & \text { if } \sum_{i=1}^{3} x_{i} \leq 0 .\end{cases}
$$

Let us assume hypothetically that agents' satisfaction functions are given by

$$
\begin{aligned}
& I_{v}^{1}\left(x_{1}\right)=\frac{x_{1}^{2}}{100}, \\
& I_{v}^{2}\left(x_{2}\right)=\frac{x_{2}^{2}}{225}, \\
& I_{v}^{3}\left(x_{3}\right)=\frac{x_{3}^{2}}{400}, \\
& P_{v}^{1}\left(y_{1}\right)=\frac{y_{1}^{2}}{78400}, \\
& P_{v}^{2}\left(y_{2}\right)=\frac{y_{2}^{2}}{93025}, \\
& P_{v}^{3}\left(y_{3}\right)=\frac{y_{3}^{2}}{115600} .
\end{aligned}
$$

Therefore, $v\left(Q_{1}\right)=20, v\left(Q_{2}\right)=45$, and $v\left(Q_{3}\right)=80$. The negotiation process goes on as follows.

Stage 1. Let the first proposal offered by the mediator be

$$
\begin{aligned}
& \left(\mathbf{x}^{1}, \mathbf{y}^{1}\right) \\
& =\left(\begin{array}{llc}
(2.22874,5) & (3.45151,10) & (4.31975,15) \\
(3.37124,6) & (4.93382,15) & (6.6949494,25) \\
(4.40002,9) & (6.61467,20) & (8.98531,40)
\end{array}\right) .
\end{aligned}
$$

The degrees of satisfactions provided by the players are

$$
\begin{aligned}
& \left(I_{v}^{i}\left(x_{i j}^{1}\right), P_{v}^{i}\left(y_{i j}^{1}\right)\right) \\
& \quad=\left(\begin{array}{ccc}
(.0496, .0002) & (.119, .0012) & (.186, .028) \\
(.050, .0001) & (.108, .0024) & (.199, .006) \\
(.0484, .0007) & (.103, .0034) & (.205, .016)
\end{array}\right) .
\end{aligned}
$$

Thus, $\operatorname{var}\left(\mathbf{x}^{1}, \mathbf{y}^{1}\right)=.0212946$.

Stage 2. Thus, the second proposal offered by the mediator is

$$
\begin{aligned}
& \left(\mathbf{x}^{2}, \mathbf{y}^{2}\right) \\
& \quad=\left(\begin{array}{lll}
(2.223,7.068) & (3.251,16.426) & (4.526,15) \\
(3.306,6.909) & (5.060,14.789) & (6.633,25) \\
(4.471,6.023) & (6.689,13.785) & (8.841,40)
\end{array}\right) .
\end{aligned}
$$

Therefore, $\operatorname{var}\left(\mathbf{x}^{2}, \mathbf{y}^{2}\right)=.012687$.
Stage 3. The third proposal offered by the mediator is

$$
\begin{aligned}
& \left(\mathbf{x}^{3}, \mathbf{y}^{3}\right) \\
& =\left(\begin{array}{ccc}
(2.219,5.413) & (3.392,11.831) & (4.388,17.429) \\
(3.356,6.690) & (4.956,14.789) & (6.687,25) \\
(4.425,7.897) & (6.652,18.380) & (8.925,37.571)
\end{array}\right) .
\end{aligned}
$$

Consequently, $\operatorname{var}\left(\mathbf{x}^{3}, \mathbf{y}^{3}\right)=.00922435$.

Thus, the variance of the approximate solutions decreases gradually at each stage of negotiation. If all the players agree to keep the variance below 0.0095 , then $\left(\mathbf{x}^{3}, \mathbf{y}^{3}\right)$ will be the solution under negotiation.

Let us recall the solution at the second stage of example 1 in [3] as follows:

$$
\begin{aligned}
\left(\mathbf{x}^{2}, \mathbf{y}^{2}\right) & \\
= & \left(\begin{array}{ccc}
(2.223,7.026) & (3.252,16.426) & (4.526,32.430) \\
(3.305,6.951) & (5.047,14.790) & (6.647,25) \\
(4.472,6.023) & (6.701,13.784) & (8.827,22.570)
\end{array}\right) .
\end{aligned}
$$

A close look at this solution reveals that players 1 and 2 are more satisfied with the solution given by the present model at stage 2 than that in [3] and the proportion of the resource investment to the payoff for player 3 is almost maintained in either model. This explains the interdependence of the two satisfaction functions each player possesses.

It is worth mentioning that the penalties of the players at the last stage $t=3$ are zero in the above example. However, the following examples show that this is not always true.

Example 2. Consider the cooperative fuzzy game $v$ with two players $P_{1}$ and $P_{2}$ and two fuzzy coalitions $S_{1}$ and $S_{2}$, defined by

$$
v\left(\left(x_{1}, x_{2}\right)\right)= \begin{cases}\frac{\left(x_{1}+x_{2}\right)^{2}}{2} & \text { if } x_{1}+x_{2} \geq 2 \\ \left(x_{1}+x_{2}\right) & \text { if } 0 \leq x_{1}+x_{2} \leq 2\end{cases}
$$

Let the available resource at the players' disposal be $R_{1}=$ 4 and $R_{2}=8$ and the sufficient resources for the coalitions $Q_{1}=6$ and $Q_{2}=6$. Let us assume hypothetically that players' satisfaction functions are given by

$$
\begin{aligned}
& I_{v}^{1}\left(x_{1}\right)=\frac{x_{1}^{2}}{16}, \\
& I_{v}^{2}\left(x_{2}\right)=\frac{x_{2}}{8}, \\
& P_{v}^{1}\left(y_{1}\right)=\frac{y_{1}}{40} \\
& P_{v}^{2}\left(y_{2}\right)=\frac{y_{2}^{2}}{4096} .
\end{aligned}
$$

Thus, $v\left(s_{1}\right)=18, v\left(s_{2}\right)=18$. The negotiation process goes on as follows: 

be

Let at some stage $t$ the proposal offered by the mediator

$$
\left(\mathbf{x}^{t}, \mathbf{y}^{t}\right)=\left(\begin{array}{ll}
(2,7.27168) & (2,7.27168) \\
(4,10.7283) & (4,10.7283)
\end{array}\right)
$$

If the negotiation stops here and $\left(\mathbf{x}^{t}, \mathbf{y}^{t}\right)$ is the required solution of the problem, using (a) to (e), we see that the penalty of player $P_{1}$ is $\sum_{j=1}^{2} p_{1}\left(x_{1 j}, y_{1 j}\right)=0+0=0$ and penalty of player $P_{2}$ is given by $\sum_{j=1}^{2} p_{2}\left(x_{2 j}, y_{2 j}\right)=0.622996+$ $0.622996=1.24599$.

\section{Conclusion}

This paper illustrates a dynamic allocation process to solve the problem of resource investment and the corresponding allocation of payoffs among the players in a cooperative environment contemporaneously. Since players may be involved in different projects at a time and are able to provide only fractions of their resources, we adopt a cooperative fuzzy game theoretic approach. Convergence in the proposed model is restrictive and each player is endowed with two satisfaction functions: one for resource investment and the other for payoff allocation. Combining these functions to have a common satisfaction function and obtaining a more general formulation for convergence are the topics of our future study.

\section{Conflicts of Interest}

The authors declare that there are no conflicts of interest regarding the publication of this paper.

\section{Acknowledgments}

The work is partially supported by UKIERI [184-15/2017(IC)]. The authors acknowledge the comments and suggestions of Rajnish Kumar and Radko Mesiar and the seminar participants of the 2013 IEEE International Conference on Fuzzy Systems (FUZZ).

\section{Endnotes}

1. Let $m$ be the maximum possible size of the fuzzy coalition structure $\mathbf{S}^{R}(m, n)$ accepted by all the players. Each player $i$ would estimate the total resource to be invested for each of the $m$ coalitions. We call this her budget; see [2]. We assume that, for the $j$ th coalition variable $\mathbf{S}_{j}^{n}(R)$, player $i$ 's total budget estimate be $Q_{i j} \leq R_{i}$. Therefore the total budget vector estimated by the $n$ players is $\left(Q_{1 j}, Q_{2 j}, \ldots, Q_{n j}\right)$. We further assume that $\mathbf{S}_{j}^{n}(R)$ can work with budget $a_{j}=\min \left\{Q_{1 j}, Q_{2 j}, \ldots, Q_{n j}\right\}$ at high risk while working with $b_{j}=\max \left\{Q_{1 j}, Q_{2 j}, \ldots, Q_{n j}\right\}$ at low risk. This assumption is intuitive as each coalition requires a sufficient amount of budget to work with and we can expect that risk level will vary according to the investment. Let $\mathbf{S}^{R}\left(m^{*}, n\right)=\left\{\mathbf{S}_{1}^{n}(R), \mathbf{S}_{2}^{n}(R), \ldots, \mathbf{S}_{m^{*}}^{n}(R)\right\}$ be an optimal coalition structure obtained by the mediator along with the corresponding budget vector $\left(Q_{1}, Q_{2}, \ldots, Q_{m^{*}}\right)$ (with regard to the cooperative fuzzy game $v$ ) such that $\sum_{j=1}^{m^{*}} v\left(Q_{j}\right)$ is maximum and $\sum_{j=1}^{m^{*}} Q_{j}=$ $R$. This task would be performed in the following three steps.

Step 1. Find all the regions of the form $\left\{a_{j} \leq Q_{j} \leq b_{j}, j=\right.$ $\left.1,2, \ldots, m^{\prime}\right\}$, where $m^{\prime} \leq n$ and $\sum_{j=1}^{m^{\prime}} a_{j} \leq \sum_{i=1}^{n} R_{i} \leq$ $\sum_{j=1}^{m^{\prime}} b_{j}$

Step 2. Solve the following optimization problem for each region obtained in Step 1:

$$
\underset{\left(Q_{1}, Q_{2}, \ldots, Q_{m^{\prime}}\right)}{\arg }\left(\max \left(\sum_{j=1}^{m^{\prime}} v\left(Q_{j}\right): a_{j} \leq Q_{j} \leq b_{j}\right)\right) .
$$

Step 3. Select those solutions $\left(Q_{1}, Q_{2}, \ldots, Q_{m^{*}}\right)$ obtained in Step 2, for which $\sum_{j=1}^{m^{*}} v\left(Q_{j}\right)$ is maximum.

\section{References}

[1] S. Borkotokey and R. Neog, "Allocating Profit Among Rational Players in a Fuzzy Coalition: A Game Theoretic Model," Group Decision and Negotiation, vol. 21, no. 4, pp. 439-459, 2012.

[2] S. Borkotokey and R. Neog, "Dynamic resource allocation in fuzzy coalitions: a game theoretic model," Fuzzy Optimization and Decision Making. A Journal of Modeling and Computation Under Uncertainty, vol. 13, no. 2, pp. 211-230, 2014.

[3] S. Borkotokey and R. Neog, "Role of satisfaction in resource accumulation and profit allocation: A fuzzy game theoretic model," in Proceedings of the 2013 IEEE International Conference on Fuzzy Systems, FUZZ-IEEE 2013, pp. 1-8, 2012.

[4] J. Yao, "Dynamic game decision about supply chain resource integration in mass customization," Journal of Intelligent \& Fuzzy Systems. Applications in Engineering and Technology, vol. 28, no. 5, pp. 2083-2093, 2015.

[5] S. C. Stubberud and K. A. Kramer, "A game theoretic sensor resource allocation using fuzzy logic," Advances in Fuzzy Systems-Applications and Theory, Article ID 792059, 2013.

[6] J. A. Filar and L. A. Petrosjan, "Dynamic cooperative games," International Game Theory Review, vol. 2, no. 1, pp. 47-65, 2000.

[7] D. Bauso and J. Timmer, "On robustness and dynamics in (un)balanced coalitional games," Automatica, vol. 48, no. 10, pp. 2592-2596, 2012.

[8] B. Ellingsæter, "Frequency allocation game in satisfaction form," Transactions on Emerging Telecommunications Technologies, vol. 25, pp. 1238-1251, 2004.

[9] Y.-H. Liao, "Fuzzy games: a complement-consistent solution, axiomatizations and dynamic approaches," Fuzzy Optimization and Decision Making. A Journal of Modeling and Computation Under Uncertainty, vol. 16, no. 3, pp. 257-268, 2017.

[10] F. Meng and X. Chen, "Cooperative fuzzy games with convex combination form," Asia-Pacific Journal of Operational Research, vol. 33, no. 1, 1650007, 25 pages, 2016.

[11] J. P. Aubin, Mathematical Methods of Game and Economic Theory, North-Holland, Amsterdam, Revised edition, 1982.

[12] D. Butnariu, "Stability and Shapley value for an n-persons fuzzy game," Fuzzy Sets and Systems, vol. 4, no. 1, pp. 63-72, 1980. 
[13] R. Branzei, D. Dimitrov, and S. Tijs, Models in Cooperative Game Theory: Crisp, Fuzzy and Multichoice Games, Lecture Notes in Economics and Mathematical Systems, Springer-Verlag, Berlin Heidelberg, New York, NY, USA, 2004.

[14] Y. Azrieli and E. Lehrer, "On some families of cooperative fuzzy games," International Journal of Game Theory, vol. 36, no. 1, pp. $1-15,2007$.

[15] M. Mares and M. Vlach, "Fuzzy coalitional structures (alternatives)," Mathware Soft Computing, vol. 13, pp. 59-70, 2006.

[16] M. Mares and M. Vlach, "Linear coalitional games and their fuzzy extensions," International Journal of Uncertainty, Fuzziness and Knowledge-Based Systems, vol. 9, pp. 341-354, 2001.

[17] M. Tsurumi, T. Tanino, and M. Inuiguchi, "A Shapley function on a class of cooperative fuzzy games," European Journal of Operational Research, vol. 129, no. 3, pp. 596-618, 2001. 


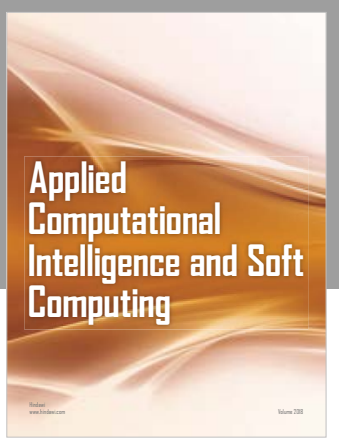

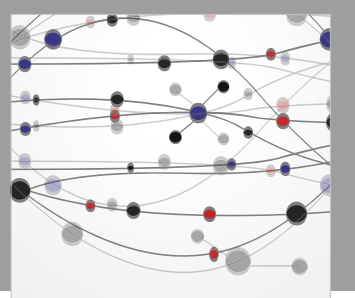

The Scientific World Journal
Submit your manuscripts at

Computing
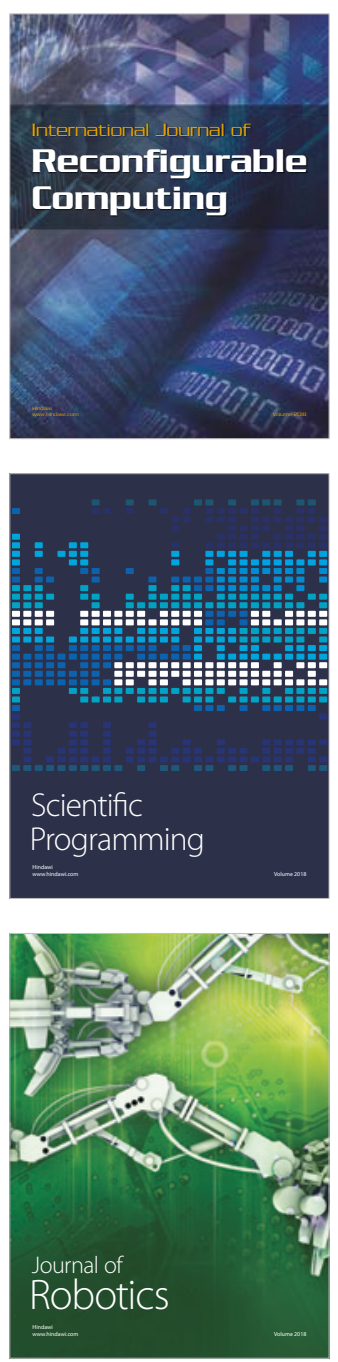

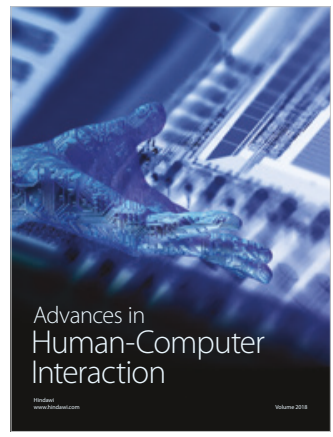

Human-Compute

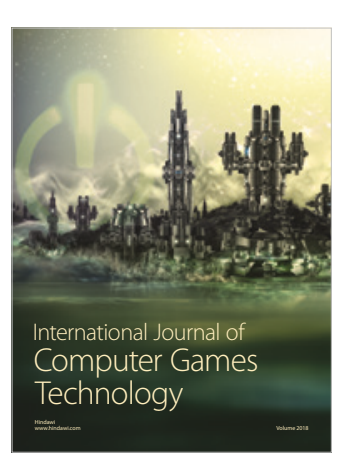

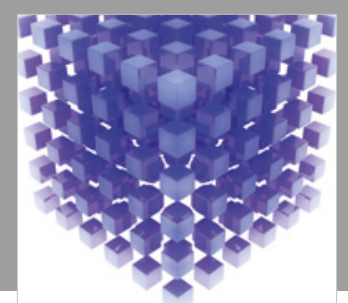

Mathematical Problems in Engineering

\section{Engincering}
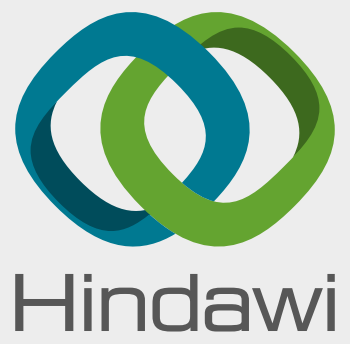

www.hindawi.com
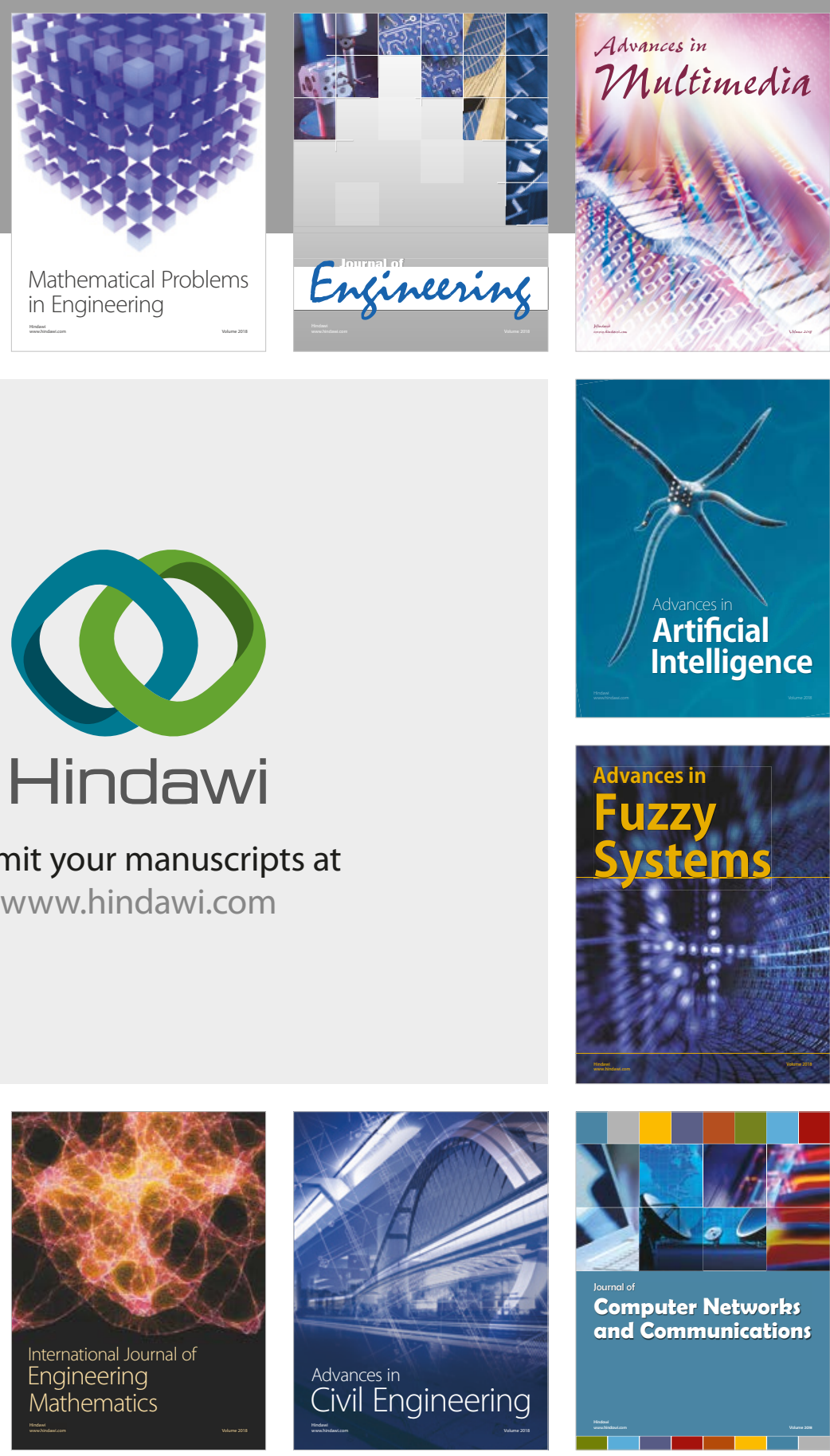

Computer Networks and Communications

Multimedia
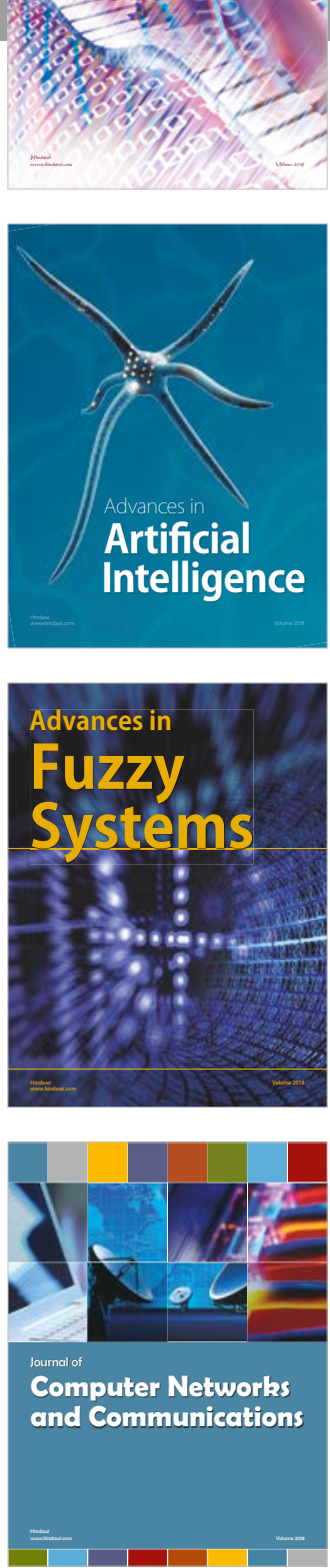

Advances in

Modelling \&

Simulation

in Engineering

interaction

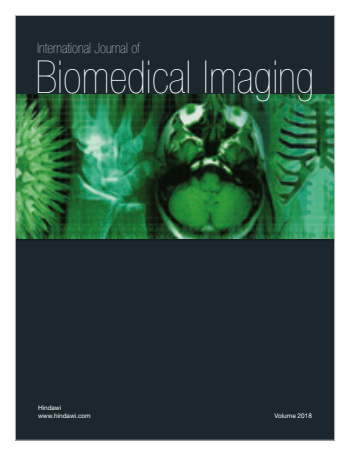

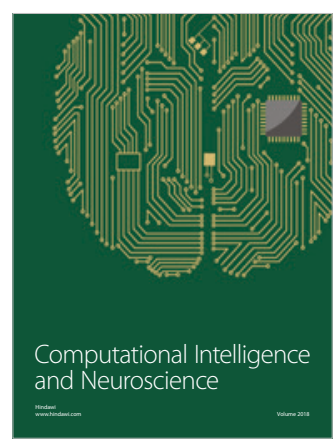

